\title{
STUDENTS EMOTIONAL STATE DURING RECREATIONAL SKIING
}

\author{
Intars Nikonovs
}

Juris Grants

Ivars Kravalis

Latvian Academy of Sport Education

\begin{abstract}
The aim of the research was to evaluate emotional state before, after and in the next day after the ski hiking. The distance was $24 \mathrm{~km}$ and lasted 8 hours. To assess the ski hiker's emotions the following 3 tests were conducted - before the ski hike, after the ski hike and 16 hours after the hike. Emotional state was set by the two different methods. One included assessing participant's dynamic of emotional state with subjective measurement using questioner, but other using subjective method by analyzing person's facial expressions. The results showed improved emotional state in both ways. Although, using objective method, improved positive emotions were more in the next day of the ski hike.
\end{abstract}

Keywords: ski hike, emotional state.

\section{Introduction}

Different kinds of recreation activities, which are helping to restore emotional levels, are becoming more and more popular. Running competitions in spring, summer and autumn periods, as well as skiing in winter attract more and more competitors and spectators. For example most popular mass skiing event in the world Vasaloppet (15 800 skiers allowed to take part) was sold out in only 80 seconds this year. Lot of the first rows skiers are professionals and skiing to be as fast as possible. Rest of them are recreational skiers and their main purpose is to be in the event, to finish and raise their emotional level. But in the same time it often happens that competitors underestimate their abilities and crushes in physically, but mainly psychologically.

Recreation activities as is our ski hiking are more effective recreation activity. There is no time hurdle in people's heads, which interfers to experiencing recreation feelings in skiing and mass running competitions. Skiing nowadays is mainly performed as competitions or as recreational activities (Bergh, 2000). If skiing is used as recreational activity it should fulfil the main function of recreations - improve emotional state (Grants, 2011).

Emotions (from Latin: emovere - to excite, thrill) are a humans and animals subjective responses to effect of internal and external irritant. Emotions are closely linked to the individual's current needs. Psychological basis of emotions is diverse and congenital self-regulation mechanisms of central nervous system 
which stereotypically reflects individual's attitude to problem situations or a specific event (Eckman \& Friesen, 2003).

English sport psychologist Lane notes, that there is not one clear definition of emotions, but agrees the theory that emotions are reaction on incentive, which is sometimes real or imagined. This definition is wide spread in field of sport psychology science (James, 1994). Definition makes connection with imagination. Human body don't separate events which are happening in real life or in imagination. Corresponding physiologic reactions takes place in both occasions Calvo \& Lunqvist, 2008).

$\mathrm{K}$. Izard has developed a theory of emotion differentiation and considers that there are 10 indigenous types of emotions - joy, interest, surprise, sorrow, anger, disgust, contempt, fear, shame, guilt (Schiano et. al., 2000). There are different theories of emotions, one of often used is theory of six basic emotions happiness, surprise, anger, fear, sadness, disgust, in the same time each emotion has universal gesture, but one emotion can include a number of mimic expressions. There is conflicting data on whether contempt, interest, shame and guilt have universal facial expressions. He also does not recognize the distribution of emotions in positive and negative, because sometimes person when feeling disgust (negative emotions) actually feels comfortable and superior over others. Others in the same time cry, when watching TV with pleasure. It means that very rarely person feels one concrete emotion; often it is complex of emotions (Eckman \& Friesen, 2003).

To assess the level of emotions mainly psychological tests are made and participant's emotional levels of participants are set on information of participant's subjective opinion. In the same time there is apparatus which can assess individual's emotions by reading face. Special program analyzes 500 points in persons face. This method allows making objective assessments of individual's emotional state. By connecting these two methods we set emotional level of ski hikers before, after and in next day after ski hike. Does emotional state after long recreational activity is raised right after ski hike our in the next day were main findings. And does participants subjective assessment of emotions are similar to objective method.

As well as rising up the theory of six basic emotions, Eckman is suggesting universal gestures for each emotion, but at the same time inferring that there are many forms of expressions. Eckman also studied what kind of gestures happen in people's face when experiencing different emotions unconciously. Different kinds of emotions have optimal zones for identification. For example emotions when feeling sad our scared are identified more easily in eye area if compared with lower area of face. Expression anger - calmness is easily set in area of forehead and eyebrows. Other expressions such as happiness, disgusted are set more often according to changes in lower face area. (Eckman, 2003)

The theories of Eckman studies used as base in the FaceReader 3.0 apparatus which we used in our study (FaceReaderTM, 2010). 
The organisation of the research was made in three stages. In preparation stage the distance and route of ski hike was planned. In second stage - testing of emotional state and retesting of emotional state 1 hour after hike and in the next day - 16 hours after the hike. In the last stage video analyses were made to set levels of emotions using FaceReader 3.0.

The distance of the ski hike was $24 \mathrm{~km}$ and it was 8 hours long. The first 3.5 hours of the ski hike were skiing, then 1 hour for rest and for lunch and again 3.5 hours of skiing. The degree of difficulty was average for the hike.

\section{Testing of emotional state}

To assess the emotional level before ski hike and determine the dynamics of those indicators after ski hike, testing of emotional state were made. The procedure was carried out using FaceReader 3.0 (developed by Noldus Information technology - Netherlands) and Sport Emotion Questioner (SEQ). The basics of FaceReader 3.0 program is live analysis of facial expressions during interview. The program consists of six basics emotions (by P. Eckman) happy, sad, angry, surprised, disgusted, scared and the state when there is no emotions in face - neutral. The testing of emotional state was made 3 times before the ski hike, 1 hour after the ski hike and next day -16 hours after the ski hike.

Survey

After each testing of emotional state ski hikers were asked to fill in the Sport Emotion questionnaire (SEQ). This includes 22 emotions and respondent have to value them in 5 levels - not at all, a little, moderately, quite a bit, extremely. After summing up all the answers, the count of points shows the level of anxiety, dejection, excitement, anger, and happiness (Jones et. al., 2005).

\section{Participants}

Seven first year master level students from Latvian Academy of Sport Education (LASE) took part in research -5 women and 2 men - aged diapason 24-35 years. The skiing experience among participants varied.

Data Analysis

Data analyses were made with data from the FaceReader 3.0 apparatus and from video analyses. To assess the dynamics of those data Student's criterion for related groups were used.

\section{Results}

The FaceReader 3.0 apparatus is made by principles of P. Eckman six base emotions theory. During the testing apparatus reads intensity of the six base emotions and the neutral state, when emotions are not read. The intensity of emotions during the test is given in form of numbers - results show the percents of test time when exact emotions were recorded. 
Dynamics of the emotional state were set by three following tests - before the ski hike, after the ski hike and 16 hours after the ski hike in the next day. As mentioned before, recreational activities should improve individual's emotional state. When using FaceReader 3.0 apparatus one of the states given is neutral. It is the state of facial expression when five basic emotions aren't so dominant. This state shows that no positive or negative emotions are read in test participant's face. By assessing this state, we can see if person became emotional. If the results show decrease in neutral state, it means that rest of emotions are dominant. In our study this connection was observed.

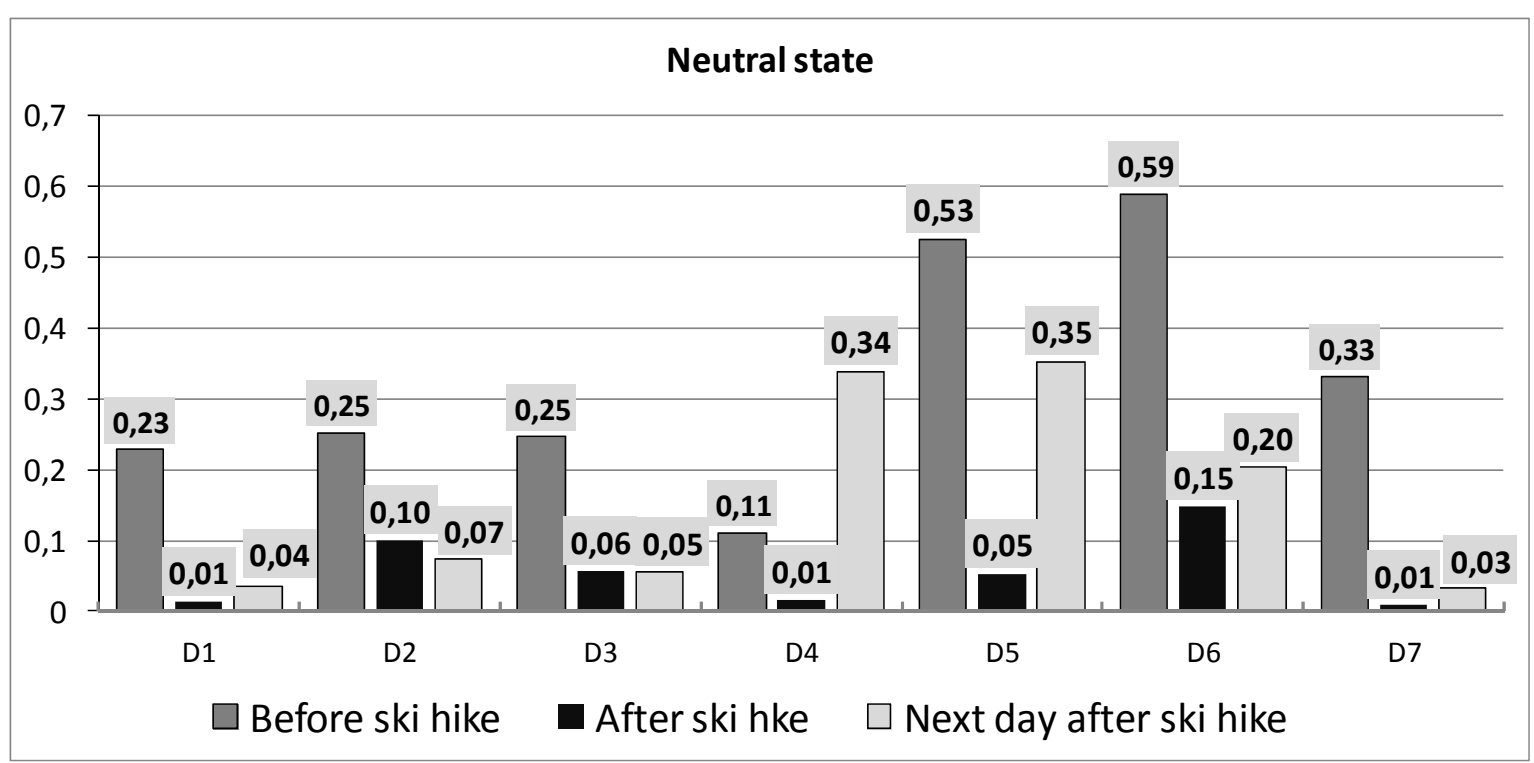

Figure 1. Dynamics of neutral state

Figure 1 shows the dynamics of emotional state - neutral. Results showed significant decrease of time when neutral state was registered. They decreased right after ski hike and were lower in testing procedure in next day after the ski hike if compared with results before hike. It is related then with effect of our ski hike as recreational activity. Participants were more emotional right after ski hike and effect maintained in next day as well. It also can be conclude that ski hike made an impact on emotional state, but from here it cannot be concluded that it was positive effect. Additional emotions are positive and negative as well so further analysis was necessary. Increased emotional state can be on negative emotions as well and that means negative effect of such kind recreational activity.

Figure 2 shows group collective average results of emotions by emotion category. Results show that before the ski hike $33 \%$ of test time participants face show neutral state - no positive or negative emotions as mentioned before. Positive emotions (happy and surprised), before the hike is $20 \%$ and $12 \%$ respectively from test time. Negative emotions (sad, angry, scared and disgusted) make $19 \%$ of the test time. In the second testing neutral state is only 
$6 \%$ of the test time - it means that mainly positive or negative emotions are mostly read. The level of positive emotions is down after the ski hike $-10 \%$ and $8 \%$ respectively of the test time. In the same time level of negative emotions is already $51 \%$ of the test time.

The third test time - 16 hours after the hike in the next day - was chosen not even to set the dynamics of emotions, but to confirm that positive emotions will be read mostly in the next day after the ski hike. As it seen the level of neutral state is $16 \%$ - almost two times lower than before hike. Emotional state happy is already $16 \%$ from the test, which is higher than immediately after hike. Results in negative emotions are lower and in two cases they are even lower than before the hike.

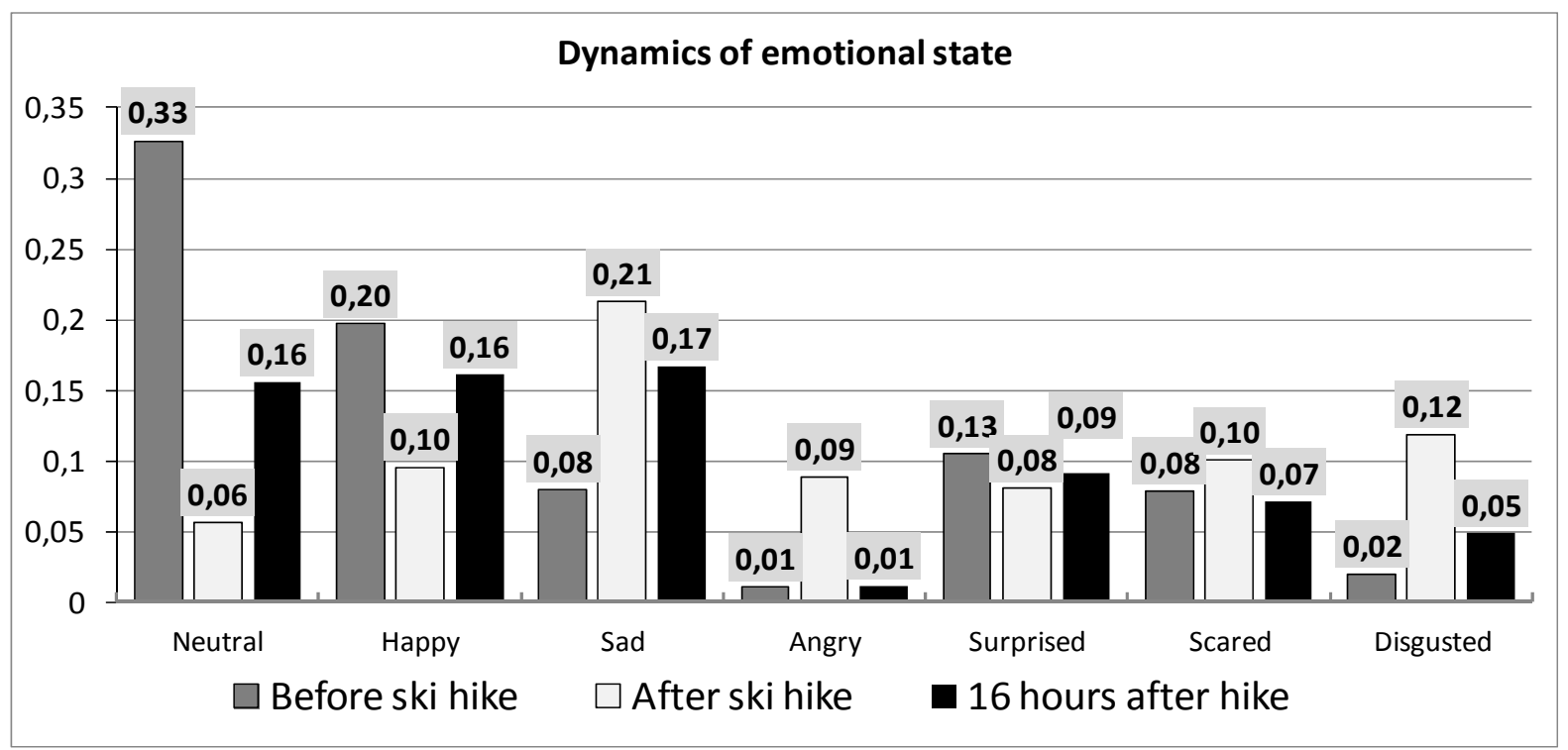

Figure 2. Dynamics of emotional state

After having sum up the results from objective emotions reading method using FaceReader 3.0, it could be concluded that right after the hike participants show more base emotions. Right after the ski hike negative emotions are in charge, but in the next day, 16 hours after the hike there is positive emotion dominance. It could be because of fatigue right after the ski hike, so in further research we suggest to test emotions during the next day. The dynamics of positive emotions show a tendency to getting higher and higher. Testing using objective method seems to prove positive effect on emotional state and positive emotions, when recreational activity is made by ski hike.

After the testing of FaceReader 3.0 participants were asked to fill in the Sport Emotion Questionnaire (SEQ), which shows the subjective assessment of emotions. The results are different from FaceReader 3.0 tests. If before the ski hike there are some signs of negative emotions, than right after the ski hike negative emotions have disappeared and the level of positive emotions are in the highest possible level. (Fig. 3) 


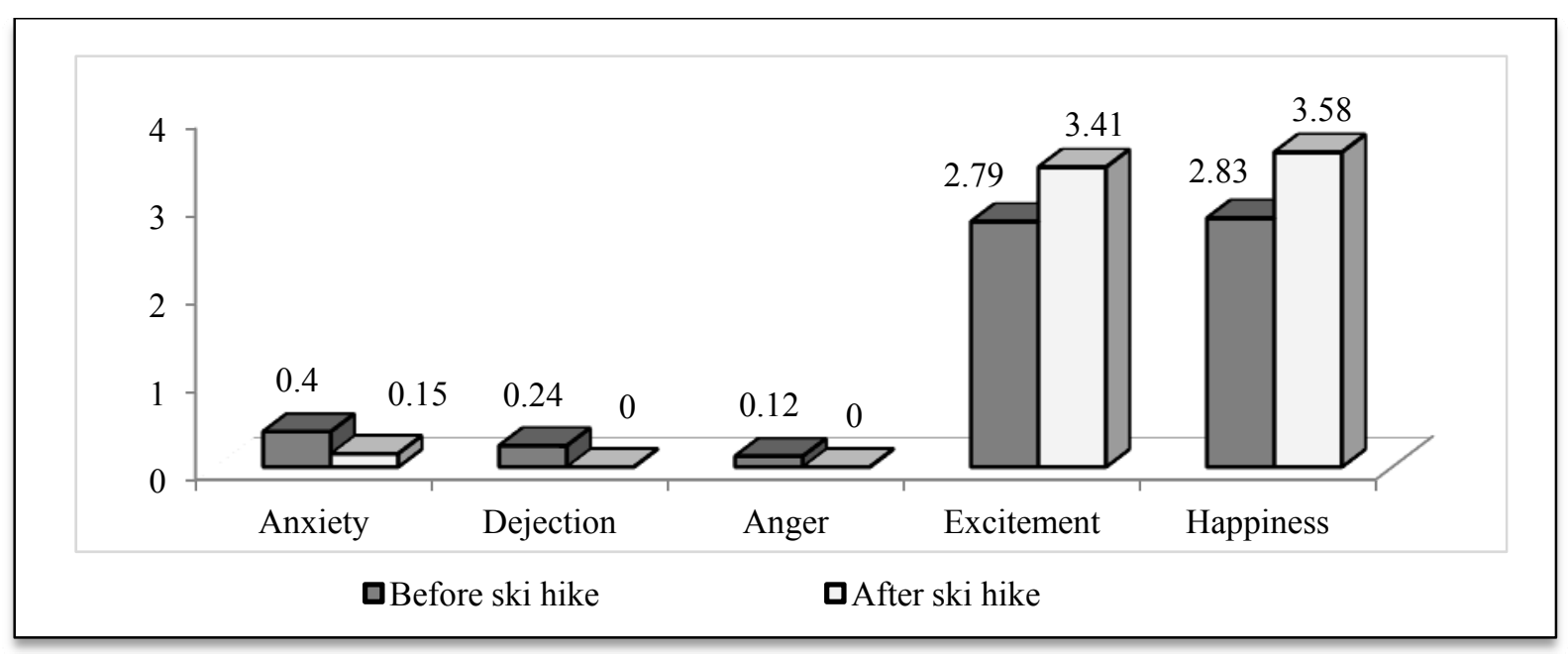

Figure 3. Subjective assessments of emotions (Sport Emotion Questionnaire)

Dynamics of emotional state shows some important things. Testing using the objective method showed an increase in level of six base emotions after hike and which means that the level of emotions increased. It happened because of increase in negative emotions although. The reason of that could be the fatigue in participants face. In the same time tests 16 hours after the ski hike showed an increase in positive emotions and decreased in negative. Subjective assessment of emotions showed positive increase of emotions right after the ski hike. So when put together both testing procedures we can conclude that such recreational activity as ski hike improved participants emotional state. Person's subjective assessment of their feeling is in our opinion the most important thing after the recreational activity. If in sport the quality criteria mainly is the result, than in recreational activities the quality criteria is improved emotional state and positive emotions.

\section{Discussion}

After summing up all the results there were few recommendations for ski hikers and ski hike organizers made. The base of recommendations is results of our research. So basically when going in ski hiking or organizing a ski hike for 6-12 people it could be helpful to take the things we recommend in mind.

After going in a prolonged ski hike (6-8 hours) positive influences on the emotional state will be seen only in next day after hike. Right after the hike, negative emotions are in dominance and to avoid this situation we recommend at the end of the hike pay greater attention to activities which could give positive effect on the emotional state. For example, 1 hour before the end of the hike includes teambuilding games, different plays our downhill ride up to situation of course.

Testing of emotional state after recreational activities isn't wide spread. One research showed similar results as our study. Research dealt with 45-55 old 
people. They were asked to go on Nordic walking, skiing or biking with intensity of $50-60 \%$ of their heart rate maximum. They tested participant's emotional state and endorphin level. Like in our research emotional state were significally improved only after 60 minutes of exercise. Main finding in this research was raised endorphin level as well. As it is known higher endorphin level indicates higher happiness (Kundzina \& Grants, 2014).

\section{Conclusions}

During the testing of emotional state the following dynamics were set:

Emotional state - Neutral - was 27\% lower after and 17\% lower 16 hours in the next day after the ski hike. Results showed that emotional levels were changed. After the ski hike there were more of 6 base emotions in ski hiker's face. One hour after the ski hike more negative emotions (sad, disgusted) were read but 16 hours afterwards these indicators are going down and more positive emotions are read. Subjective assessment of emotional state (Sport Emotions Questionnaire (SEQ)) shows positive influence on the ski hiker's emotions negative emotions are reduced and positive emotions raised.

Our research showed positive effect on increased emotional state after ski hike. Both testing methods showed that ski hike is very appropriate recreational activity and emotional state will be improved. In the same time it is always good to remember about good planning and suitable pace.

\section{References}

Bergh, U. (2000). Cross-country ski racing. In: Endurance in sport.Volume 2 of the encyclopedia of sports medicine. Blackwell science. Oxford UK.

Calvo, G.M. \& Lunqvist, D. (2008). Facial expressions of emotion (KDEF): Identification under different display - duration conditions. Behavior Research methods, 40 (1), 109115 .

Ekman, P. \& Friesen, V.W. (2003). Unmasking the face. - Malor books. Cambridge MA.

FaceReaderTM. (2010). Tool for automatic analysis of facial expression. Reference Manual Version 3. By Noldus Information Technology.

Grants, J. (2011). Let us learn skiing! Riga, LV. "Alamande” Print Agency.

Jones, M. V., Lane, A. M., Bray, S. R., Uphill, M., \& Catlin, J. (2005). Development and validation of the sport emotion questionnaire. Journal of Sports and Exercise Psychology, 27, 407-431

Kundzina, I., Grants, J. Outdoor recreation and well-being of 45-55 years old people. LASE Journal of Sport Science, 2014, Vol.5, No.2.

Russell, A. James (1994). Is there universal recognition of emotion from facial expression? A review of the cross-cultural studies: Psychological bulletin, Vol. 115, No. 1, 102-141

Schiano, J. Diane, Ehrlich Sheryl, Rahardja, Krisnawan, Sheridan, Kyle (2000). Measuring and modeling facial affect: Behavior research methods. Instruments \& Computers, 32 (4). 505-514 